\title{
Management of Pregnancy in Patients with Congenital Heart Disease
}

\author{
lan S. Harris, M.D. ${ }^{1,2,{ }^{*}}$ \\ ${ }^{1}$ Department of Medicine, Division of Cardiology, University of California, San Francisco, CA \\ 94143-0214 \\ ${ }^{2}$ Cardiovascular Research Institute, University of California, San Francisco, CA 94143-0214
}

\section{Abstract}

Congenital heart diseases are the most common birth defects in humans, affecting approximately $0.8 \%$ of all live births. In the past, many of the more severe defects resulted in profound disability and death during childhood, and adult survival was exceptional. The past 4 decades have seen dramatic improvements in the survival and quality of life of patients with the more severe defects. As a result of these improvements, the challenges of caring for adults with congenital heart disease are only now being realized. The majority of women with congenital heart disease are now expected to reach childbearing age and maternal cardiac disease is the major cause of maternal morbidity and mortality. As such, appropriate pre-pregnancy counseling and management during pregnancy are fundamental components of the care of these patients. This article describes the circulatory changes that occur during normal pregnancy and delivery, addresses the risks posed during pregnancy by specific congenital lesions, and reviews the current data on pregnancy outcomes in patients with individual congenital defects.

\section{Introduction}

Congenital heart diseases (CHD) are the most common birth defects in humans, affecting approximately $0.8 \%$ of all live births (1). The spectrum of defects is broad, ranging from complex defects that result in profound disability and death in infancy to minor defects that are discovered later in life in asymptomatic adults. Improved imaging and diagnostic methods in recent years have led to an increase in the detection and characterization of some of the more minor defects, and advances in medical and surgical care have resulted in a vast improvement in the survival and quality of life of those patients with the more severe defects. As a result of these technological innovations, adults with CHD now outnumber children with CHD in the United States (2). This improved survival has created new challenges for adult cardiologists, who are now faced with managing these patients as they develop acquired conditions of adulthood. Specifically, many women with CHD are now reaching reproductive age and maternal cardiac disease remains the largest contributor to maternal morbidity and mortality worldwide. A critical component of the care of women of reproductive age involves knowledgeable preconception counseling and skillful

\footnotetext{
(C) 2010 Elsevier Ltd. All rights reserved.

*Corresponding Author: 505 Parnassus Avenue, M314, San Francisco, CA 94143-0124 harrisi@ medicine.ucsf.edu.
}

Publisher's Disclaimer: This is a PDF file of an unedited manuscript that has been accepted for publication. As a service to our customers we are providing this early version of the manuscript. The manuscript will undergo copyediting, typesetting, and review of the resulting proof before it is published in its final citable form. Please note that during the production process errors may be discovered which could affect the content, and all legal disclaimers that apply to the journal pertain. 
management throughout pregnancy and delivery (3). Such care requires both familiarity with the congenital lesions and close collaboration with high-risk obstetrical services.

\section{Normal cardiovascular adaptations to pregnancy}

Over the course of a normal pregnancy, profound hemodynamic changes occur (Figure 1). Chief among these changes are increases in blood volume, cardiac output, left ventricular (LV) stroke work, and oxygen consumption. While these changes are well documented, their specific causes are incompletely understood.

\section{A. First and second trimester}

A reduction in peripheral vascular resistance and an increase in plasma volume are seen as early as six weeks of gestation and are associated with activation of the renin-angiotensinaldosterone system and a mild reduction of plasma atrial natriuretic peptide (ANP) concentration (4). This fall in vascular resistance is augmented after full placentation, when low placental vascular resistance allows for high uterine blood flow (5). The blood volume continues to rise over the course of the first two trimesters and reaches a plateau of 140$150 \%$ of the pre-pregnancy level at approximately 32 weeks of gestation. Concomitant with this increase in plasma volume and fall in vascular resistance, the cardiac output increases steadily until approximately 25 weeks of gestation. During the earlier stages of pregnancy, this increase is mediated primarily by an increase in the stroke volume. During later stages, an increase in heart rate is primarily responsible for the increase in cardiac output. Systolic and diastolic arterial pressures both fall, but there is a larger proportional fall in the diastolic pressure and consequently an increase in pulse pressure.

\section{B. Third trimester and term}

As noted above, the stroke volume rises until approximately 25 weeks and the blood volume reaches a plateau at approximately 32 weeks. The third trimester of pregnancy is characterized by a rapid growth in the size of the fetus, and enlargement of the uterus frequently results in hemodynamically significant compression of the inferior vena cava and a resultant drop in venous return to the heart and in cardiac output. This typically occurs when the patient is in the supine position, and rolling the patient from the supine to the left lateral position has been shown to result in an increase of $25-30 \%$ in the stroke volume and cardiac output $(5,6)$.

\section{Labor and peripartum}

During active labor, further hemodynamic changes occur. The cardiac output typically increases by an additional 50\%. Each uterine contraction forces an additional $300-500 \mathrm{ml}$ of venous blood back into the central venous system. In addition, during the second stage of labor, blood pressure and heart rate can increase markedly with pain while Valsalva maneuvers during active labor can result in large fluctuations in central venous pressure. These latter changes can be attenuated by the use of neuraxial blockade and through the use of vacuum- or forceps-assisted vaginal delivery $(7,8)$. While caesarian delivery can eliminate the hemodynamic stresses during active labor and can afford control over the timing of delivery, it is often associated with greater blood loss, and consequently greater fluid shifts, than is vaginal delivery. As such, with few exceptions, the recommendation for caesarian delivery is made on obstetric, rather than cardiologic considerations. Immediately after delivery of the placenta, splanchnic vasoconstriction occurs, and approximately $500 \mathrm{ml}$ of blood is diverted from the uteroplacental bed back into the maternal circulation. This "autotransfusion" phenomenon, in conjunction with the release of caval compression, increases central venous pressures, ventricular preload and cardiac output and may offset the effect of peripartum blood loss. 


\section{Postpartum}

Starting at approximately 48 hours after delivery, a diuresis and natriuresis occur, and the blood volume, peripheral vascular resistance, and cardiac output return to their prepregnancy levels over the course of four to 12 weeks (9).

\section{Assessment of maternal and fetal risk and preconception counseling}

Ideally, all women of reproductive age with known CHD should undergo thoughtful evaluation prior to becoming pregnant. This evaluation should focus first on identifying and quantifying the risk to the mother. Second, it should address potential risks to the fetus, including the risk of inheriting a congenital heart defect.

\section{E. Clinical assessment}

A careful clinical assessment is essential in order to estimate the maternal and fetal risk of pregnancy. The primary focus of this assessment should be to evaluate the patient's ability to tolerate the hemodynamic changes described above. A small number of conditions are known to place women at such high risk for pregnancy-related morbidity and mortality that they should be regarded as absolute contraindications to pregnancy (Table 1). These diagnoses should result in a strong recommendation for definitive contraceptive measures and, in the case of unintended pregnancy, early termination should be offered and discussed. The presence of residual hemodynamic lesions or sequelae of palliative procedures that would warrant surgical or catheter-based intervention in the absence of pregnancy should prompt the physician to recommend deferral of pregnancy until these lesions are addressed. In the absence of any of these high-risk lesions, a more detailed evaluation can allow for risk stratification. A risk assessment tool has been proposed by Siu, et al which screens for highrisk historical features, including left heart obstruction, systemic ventricular systolic dysfunction (EF <0.4), NYHA Class > II, and prior cardiac event or arrhythmia (10). A simplified approach involves a basic functional assessment. In general, women with NYHA class I or II have a relatively favorable prognosis during pregnancy while those with more significant functional limitations have a higher risk of cardiovascular complications and should be advised against pregnancy $(3,11)$. In sedentary patients, formal exercise testing may provide additional data helpful for risk stratification. Irrespective of the initial risk estimation, care should be individualized, since functional deterioration is common during pregnancy $(12,13)$

\section{F. Estimation of fetal risk, genetic counseling and testing}

Risks of fetal heart defects are very high, and as many as $30 \%$ of all miscarriages are due to fetal CHD. Routine counseling should include an inquiry about exposure to teratogens and non-cardiac diagnoses associated with a risk for CHD (Lupus, phenylketonuria). It has been long recognized that CHDs tend to cluster in families. The recurrence risk varies by lesion, but is generally believed to be $5 \%$ to $6 \%$ for children born to women with CHD and $2 \%$ to $3 \%$ for children born to men with CHD (14). While most CHD is believed to be sporadic, $3 \%$ to 5\% of CHDs are associated with recognizable genetic syndromes (15). The genetics of congenital cardiac disease are still incompletely understood, but insights from new research in developmental biology are beginning to allow us to build an etiologic model of CHD. As this occurs, the role of genetic testing will continue to evolve. Currently, referral to a geneticist for counseling and/or testing should be considered in patients with a suspected syndromic CHD and in patients with other affected family members.

\section{G. Fetal echocardiography}

Fetal echocardiography has become a powerful tool in the detection of fetal cardiac anomalies. Prenatal detection of CHD may reduce perinatal mortality and allow for an 
opportunity to discuss the diagnosis in advance and to plan for delivery (16). Suggested indications for fetal echocardiography include familial or syndromic congenital heart disease in the parents, an abnormal level II ultrasound (a targeted, anatomical scan usually performed because of an abnormal screening ultrasound or suspected anatomic abnormality) and the presence of polyhydramnios (17).

\section{Specific conditions}

\section{H. Acyanotic heart defects without shunt}

Congenital aortic stenosis is a common congenital lesion that may escape detection in childhood. Obstruction to aortic outflow results in LV hypertrophy and "preload dependence", which may cause problems in accommodating the volume shifts of pregnancy. Furthermore, fixed LV outflow obstruction limits the patient's ability to augment cardiac output as needed in pregnancy. Early reports have suggested high risk of maternal and fetal complications during pregnancy. However, there is a broad spectrum of severity of stenosis, and risk varies accordingly. A recent study of a national database identified 35 women with congenital aortic stenosis who had a total of 58 pregnancies, of which 53 were successful (18). Cardiac complications occurred in $9.4 \%$ of pregnancies, with heart failure and atrial arrhythmias being the most common problems. Obstetric and perinatal complications occurred more frequently (in $22.6 \%$ and $24.5 \%$, respectively), and premature delivery and small-for-gestational age births were common (13.2\%). Importantly, $77.1 \%$ of the women in the study had had repair of their aortic obstruction and 41 of the 53 patients $(82 \%)$ had mild or moderate aortic stenosis at the time of pregnancy. Women with severe aortic stenosis were significantly more likely to develop heart failure during their pregnancy and to develop premature labor, a finding in agreement with earlier studies (10). In addition, women with moderate or severe aortic stenosis or NYHA class II symptoms are at greater risk for late cardiac complications after pregnancy including heart failure, arrhythmia, cardiac intervention, and cardiac death than are age- and lesion-matched women who were never pregnant (19). While data from prospective studies are lacking, these results warrant careful pre-pregnancy evaluation and counseling of patients with congenital aortic stenosis. In cases of symptomatic, severe aortic stenosis presenting during pregnancy, balloon aortic valvuloplasty in the second trimester often offers effective palliation as long as the valve is pliable and no more than mildly regurgitant $(20,21)$.

Pulmonic stenosis is relatively uncommon as an isolated congenital defect, occurring in approximately 5/10,000 live births and accounting for 2\% of CHD (Jackson 1996). Many patients remain asymptomatic throughout childhood and early adulthood. Because right ventricular (RV) outflow obstruction results in RV hypertrophy, patients may be sensitive to the volume overload that occurs during pregnancy and may be expected to have a risk for atrial arrhythmias. However, most case reports have suggested that pregnancy is well tolerated, even in patients with severe stenosis. The largest report on pregnancy in women with isolated pulmonic stenosis studied a national database and identified 51 women who had 108 pregnancies, of which 81 were completed (22). In this population, cardiac complications were uncommon, with only two cases of worsening functional status and nine cases of palpitations, none of which was investigated or found to be associated with documented arrhythmias (22). There was, however, an increased risk of miscarriage (19.4\%) and pre-term birth (16\%), and the incidence of CHD among the offspring was $3.7 \%$. Importantly, all patients had NYHA I or II functional status, 78\% had less than moderate stenosis and only one patient had severe pulmonic stenosis before pregnancy. Based on these results, the risk associated with pregnancy appears to be low in asymptomatic patients and those with less than severe pulmonic stenosis. 
Coarctation of the aorta results in upper extremity hypertension, which may be difficult to control during pregnancy (23). Excessive blood pressure lowering in the upper extremities may result in hypotension distal to the obstruction and uterine hypoperfusion. As a consequence, hemodynamically significant coarctation should be repaired before pregnancy is contemplated (24). In addition, coarctation is associated with an aortopathy that predisposes to aortic dissection. While there are no recent large-scale studies, the older literature suggests that aortic rupture is the most common cause of death in pregnant women with coarctation $(23,25)$. In light of the potential risks of aortic complications, elective caesarian section should be considered in patients with an unrepaired coarctation and enlargement of the proximal aorta (24).

\section{Acyanotic heart defects with shunt}

Atrial septal defect (ASD) is one of the most common congenital defects seen in pregnant women. Most patients are asymptomatic before pregnancy, but the chronic left-to-right shunt results in RV volume overload and enlargement, which may be exacerbated by the volume retention of pregnancy. Furthermore, right atrial enlargement may predispose to atrial arrhythmias. In a large, multinational retrospective cohort study, 67 women with unrepaired ASD and 31 women with repaired ASD had a total of 188 pregnancies. The incidence of cardiac complications was comparable between the two groups, with $4.3 \%$ experiencing arrhythmias and persistent decline in NYHA functional status in 3\%. Compared to the general population, women with an unrepaired ASD had a higher risk of pre-eclampsia (OR $=3.54,95 \%$ CI 1.26-9.98), fetal mortality (OR 5.55, 95\% CI 1.77-17.4) and small for gestational age birth (OR 1.95, 95\%CI 1.15-3.30). There was no significantly increased risk of fetal complications in patients with repaired ASDs (26).

Ventricular septal defect (VSD) is commonly encountered as an isolated defect. As a rule, large defects lead to significant LV volume overload and heart failure early in life and are generally repaired in childhood. As a result, most unrepaired defects encountered in the adult population are small. In a large, multicenter retrospective cohort study, eighty-eight women were identified who had experienced 202 pregnancies, including 46 miscarriages and nine terminations of pregnancy. 104 completed pregnancies occurred in patients with unrepaired VSD, while 43 occurred in patients with repaired VSD (27). The only cardiac complication was a single case of infective endocarditis that occurred in a patient with an unrepaired membranous VSD. Pre-eclampsia was more common in women with an unrepaired VSD compared with controls (OR 4.59, 95\% CI 2.01-10.5). Interestingly, women with repaired VSD had a higher risk of premature labor (OR 4.02, 95\% CI 1.1214.4) than women with unrepaired VSD (27). Taken together, these data suggest that isolated VSD imposes a low risk for cardiac complications during pregnancy.

Irrespective of the underlying lesion, Eisenmenger syndrome poses a high risk to the mother and the fetus, with a reported maternal death rate of $40-50 \%$ and a miscarriage rate of $30 \%$ (28). Patients should be counseled strongly not to become pregnant and a nonhormonal birth control option should be offered. If pregnancy occurs, elective early-term termination should be offered. Patients who elect to continue pregnancy should be monitored very carefully. Bed rest and gentle diuresis for right heart failure may be necessary. The systemic vasodilation of pregnancy may increase the magnitude of the rightto-left shunt. Measures to counteract this effect include supplemental oxygen and pulmonary vasodilators. However, there are no data to prove that these measures reduce the risk of death. Anticoagulation with heparin is often prescribed because of the risk for pulmonary arterial thrombosis. However, the benefit of this intervention must be weighed against the risk of pulmonary hemorrhage. 


\section{J. Cyanotic heart defects}

Cyanotic heart defects represent an anatomic and physiologic spectrum. As a whole, they pose moderate risks to the mother and the fetus during pregnancy. The overall maternal mortality among patients with unrepaired cyanotic heart disease is $2 \%$ and cardiac complications, including arrhythmias, heart failure, and infective endocarditis have been reported in 30\% of patients (29). Patients with chronic cyanosis are at risk for both thrombotic and hemorrhagic complications. Ensuring proper hydration throughout pregnancy and the use of compression stockings may mitigate thrombotic complications. Prophylactic anticoagulation is reasonable in the puerperium (24). Chronic cyanosis also results in a markedly increased risk for fetal complications including spontaneous abortion (50\%), premature delivery (30-50\%), and small for gestational age birth (24).

The Tetralogy of Fallot (TOF) is the most common cyanotic congenital cardiac defect in humans. The vast majority of adult patients with TOF will have had intracardiac repair consisting of a ventricular septal defect patch and enlargement of the RV infundibulum. This latter procedure is associated with a high incidence of pulmonic insufficiency, particularly when a transannular patch is used. This results in RV volume overload and enlargement which may confer an increased risk of right heart failure and arrhythmias during pregnancy (30). A study of 112 pregnancies in 42 women with TOF suggested generally favorable outcomes (31). There were no maternal deaths, only one premature delivery, and 6 patients with repaired TOF (7\%) reported cardiovascular complications during pregnancy. On the other hand, the rate of miscarriage was high: only 82 of the pregnancies were successful and 5 live-born infants $(6 \%)$ were found to have congenital anomalies. Two smaller studies revealed similar results (32-34). Observational studies have suggested that maternal and fetal risks are highest in patients with severe pulmonic regurgitation and with RV systolic dysfunction. If possible, these patients should be evaluated completely before pregnancy and pregnancy should be delayed if pulmonic valve replacement is indicated (35).

Until recently, complete (d-) transposition of the great vessels was most often palliated by means of an atrial switch (Mustard or Senning) procedure. This corrects the circulation and, in the absence of a persistent shunt, eliminates cyanosis. However, it leaves the morphologic $\mathrm{RV}$ as the pulmonary venous ventricle in the systemic position, creating a risk of systolic dysfunction and inadequate contractile reserve during pregnancy. Furthermore, atrial suture lines may serve as a substrate for arrhythmias. A large study of a national registry identified 28 patients who had a total of 69 pregnancies, of which 49 were completed (36). In this cohort, the most common cardiovascular complication during pregnancy was clinically significant arrhythmia, occurring in $22 \%$ of cases. A prior history of arrhythmia was associated with a greater risk of arrhythmia during pregnancy. While there were no maternal deaths, heart failure occurred during two pregnancies in two patients and deterioration of NYHA class was found during $17(34.7 \%)$ pregnancies. This decline in functional status persisted for more than a year postpartum in four patients (8.2\%). Obstetrical complications including premature rupture of membranes, prolonged second stage of labor, and postpartum hemorrhage were common and combined fetal and neonatal mortality was $11.8 \%$ (36). To date, there are insufficient data concerning pregnancy outcomes in patients with d-TGA who have undergone arterial switch procedures, although most experts suggest a prepregnancy evaluation to exclude myocardial ischemia, supravalvular obstruction, and dysfunction of the neo-aortic valve (37).

The Fontan operation was introduced as a palliative procedure for patients with tricuspid atresia and has since been used extensively to address a range of defects characterized by a single functional ventricle. The basic Fontan repair directs systemic venous blood into the pulmonary artery such that it bypasses the right heart. Systemic venous pressures are therefore elevated and equal to mean pulmonary arterial pressure. Patients with Fontan 
physiology are typically sensitive to preload changes and are at risk for edema, ascites, and arrhythmias during pregnancy. Several small case series have been reported in the literature with generally favorable results. A study employing a national registry in the Netherlands identified 10 pregnancies in six patients (38). Of these, there were 5 miscarriages and one aborted ectopic pregnancy. Of the four successful pregnancies, two were complicated by a decline in functional status and one was complicated by atrial arrhythmias. In addition, infertility, sub-fertility, and menstrual disorders were common in this cohort. Importantly, the patients reported in this study had good ventricular systolic function and normally functioning Fontan circuits. These findings were compatible with earlier multicenter reports (39). Because of the unusual hemodynamics associated with Fontan physiology, delivery and peripartum management should be performed in a center with significant experience in the medical, obstetric, and anesthesiologic care of these patients. In our center, we have had excellent results from forceps-assisted vaginal delivery performed under continuous spinal anesthesia.

\section{K. Aortic diseases}

Several genetic disorders involving the aorta have been reported to result in a risk of aortic dissection or rupture during pregnancy. Chief among these are Marfan syndrome, the vascular subtype (IV) of Ehlers-Danlos syndrome, and the Loeys-Dietz syndrome, a recently recognized familial thoracic aortic aneurysm syndrome caused by a mutation in the tgf- $\beta$ gene with autosomal dominant inheritance. Patients with Marfan syndrome and an aortic root diameter of $>4.0 \mathrm{~cm}$ have a $10 \%$ risk of dissection during pregnancy while those with normal aortic root diameters have a risk for dissection of approximately $1 \%(40,41)$. Current guidelines suggest elective surgical repair before pregnancy for women with aortic root dimension greater than $4.7 \mathrm{~cm}$ (24). Women who elect to proceed with pregnancy should be followed closely with surveillance echocardiography. Early epidural anesthesia and assisted second stage of labor are recommended in patients with aortic root diameter < $4.5 \mathrm{~cm}$, bearing in mind the increased incidence of dural ectasia in these patients. Elective caesarian delivery is recommended for patients with aortic root diameter $>=4.5 \mathrm{~cm}$ (24). In contrast to those with Marfan syndrome, patients with Loeys-Dietz syndrome appear to be at risk for dissection even in the absence of prior progressive aortic enlargement. While successful pregnancy and delivery have been reported (42), the absolute risk remains unclear and at this point, most experts consider the Loeys-Dietz syndrome to be a contraindication to pregnancy.

\section{Special considerations}

\section{Anticoagulation}

The use of warfarin during the first trimester has been associated with a risk for embryopathy, although the absolute incidence is unknown. The risk does appear to be doserelated, with a very low incidence in patients taking $5 \mathrm{mg}$ per day or less (43). Multiple studies have shown that heparin therapy is associated with a higher incidence of thrombotic complications during pregnancy (44) and that low-dose warfarin use throughout pregnancy is the safest approach from the maternal perspective. While the risks and benefits of various regimens must be discussed with the patient, a rational alternative is to switch from warfarin to subcutaneous unfractionated heparin before the sixth week of gestation and to resume warfarin after the twelfth week (45). Heparin should be restarted at the $36^{\text {th }}$ week of gestation to minimize the risk of fetal intracranial hemorrhage during delivery (44). Alternatively, elective caesarian section can be planned for 36 weeks and heparin can be held four hours before surgery (45). 


\section{Endocarditis prophylaxis}

The risk of infective endocarditis following vaginal delivery is very low. The ACC/AHA guidelines governing the use of prophylactic antibiotics were revised in 2007 and, while decisions must be tailored to the individual patient, the guidelines no longer formally support the routine use of antibiotics at the time of vaginal delivery irrespective of the cardiac lesion (46). The indications for prophylactic antibiotics at the time of dental procedures during pregnancy are the same as in the non-pregnant state and are discussed elsewhere in this issue. Recommendations for routine antibiotics after a skin incision for caesarian section are unchanged.

\section{Conclusion}

Recent advances have led to improved survival and function in patients with CHD and to an increase in the population of reproductive-age women with CHD. As our experience in managing these patients during pregnancy has grown, we have learned that the majority of patients with CHD can expect to tolerate pregnancy and delivery with acceptably low risk for complications. With this realization comes the responsibility to discuss these issues directly and frankly with our patients and an opportunity to reach decisions of care that are sensitive to patient preferences and acknowledgment of risks. At the same time, it is clear that further research is necessary, particularly in the form of prospective studies, to refine risk prediction and clinical decision-making and to determine optimal management of patients with CHD during pregnancy and labor.

\section{List of Abbreviations}

$\begin{array}{ll}\text { ASD } & \text { Atrial Septal Defect } \\ \text { ANP } & \text { Atrial Natriuretic Peptide } \\ \text { CHD } & \text { Congenital Heart Disease } \\ \text { CI } & \text { Confidence Interval } \\ \text { D-TGA } & \text { d-Transposition of the Great } \\ & \text { Great Arteries/Vessels) } \\ \text { EF } & \text { Ejection Fraction } \\ \text { LV } & \text { Left Ventricle } \\ \text { NYHA } & \text { New York Heart Association } \\ \text { OR } & \text { Odds Ratio } \\ \text { RV } & \text { Right Ventricle } \\ \text { TOF } & \text { Tetralogy of Fallot } \\ \text { VSD } & \text { Ventricular Septal Defect }\end{array}$

\section{References}

1. Hoffman JI, Kaplan S. The incidence of congenital heart disease. J Am Coll Cardiol 2002;39(12): 1890-1900. [PubMed: 12084585]

2. Hoffman JI, Kaplan S, Liberthson RR. Prevalence of congenital heart disease. Am Heart J 2004;147(3):425-439. [PubMed: 14999190]

3. Cardiac disease in pregnancy. ACOG technical bulletin number 168--June 1992. Int J Gynaecol Obstet 1993;41(3):298-306. [PubMed: 8102996] 
4. Chapman AB, Abraham WT, Zamudio S, Coffin C, Merouani A, Young D, et al. Temporal relationships between hormonal and hemodynamic changes in early human pregnancy. Kidney Int 1998;54(6):2056-2063. [PubMed: 9853271]

5. Fujitani S, Baldisseri MR. Hemodynamic assessment in a pregnant and peripartum patient. Crit Care Med 2005;33 Suppl(10):S354-S361. [PubMed: 16215359]

6. Clark SL, Cotton DB, Pivarnik JM, Lee W, Hankins GD, Benedetti TJ, et al. Position change and central hemodynamic profile during normal third-trimester pregnancy and post partum. Am J Obstet Gynecol 1991;164(3):883-887. [PubMed: 2003555]

7. Gerhardt MA, Gunka VB, Miller RJ. Hemodynamic stability during labor and delivery with continuous epidural infusion. J Am Osteopath Assoc 2006;106(12):692-698. [PubMed: 17242413]

8. Van de Velde M, Van Schoubroeck D, Jani J, Teunkens A, Missant C, Deprest J. Combined spinalepidural anesthesia for cesarean delivery: dose-dependent effects of hyperbaric bupivacaine on maternal hemodynamics. Anesth Analg 2006;103(1):187-190. table of contents. [PubMed: 16790651]

9. Capeless EL, Clapp JF. When do cardiovascular parameters return to their preconception values? Am J Obstet Gynecol 1991;165(4 Pt 1):883-886. [PubMed: 1951547]

10. Siu SC, Sermer M, Colman JM, Alvarez AN, Mercier LA, Morton BC, et al. Prospective multicenter study of pregnancy outcomes in women with heart disease. Circulation 2001;104(5): 515-521. [PubMed: 11479246]

11. ACOG practice bulletin. Medical management of endometriosis. Number 11, December 1999 (replaces Technical Bulletin Number 184, September 1993). Clinical management guidelines for obstetrician-gynecologists. Int J Gynaecol Obstet 2000;71(2):183-196. [PubMed: 11186465]

12. Szekely P, Turner R, Snaith L. Pregnancy and the changing pattern of rheumatic heart disease. $\mathrm{Br}$ Heart J 1973;35(12):1293-1303. PMCID: 458797. [PubMed: 4759927]

13. Uebing A, Arvanitis P, Li W, Diller GP, Babu-Narayan SV, Okonko D, et al. Effect of pregnancy on clinical status and ventricular function in women with heart disease. Int J Cardiol 2010;139(1): 50-59. [PubMed: 18835051]

14. Hoffman JI. Incidence of congenital heart disease: II. Prenatal incidence. Pediatr Cardiol 1995;16(4):155-165. [PubMed: 7567659]

15. Meberg A, Otterstad JE, Froland G, Lindberg H, Sorland SJ. Outcome of congenital heart defects-a population-based study. Acta Paediatr 2000;89(11):1344-1351. [PubMed: 11106048]

16. Saari-Kemppainen A, Karjalainen O, Ylostalo P, Heinonen OP. Ultrasound screening and perinatal mortality: controlled trial of systematic one-stage screening in pregnancy. The Helsinki Ultrasound Trial. Lancet 1990;336(8712):387-391. [PubMed: 1974940]

17. Sharony R, Fejgin MD, Biron-Shental T, Hershko-Klement A, Amiel A, Levi A. Who should be offered fetal echocardiography? One center's experience with 3965 cases. Isr Med Assoc J 2009;11(9):542-545. [PubMed: 19960848]

18. Yap SC, Drenthen W, Pieper PG, Moons P, Mulder BJ, Mostert B, et al. Risk of complications during pregnancy in women with congenital aortic stenosis. Int J Cardiol 2008;126(2):240-246. [PubMed: 17482293]

19. Tzemos N, Silversides CK, Colman JM, Therrien J, Webb GD, Mason J, et al. Late cardiac outcomes after pregnancy in women with congenital aortic stenosis. Am Heart J 2009;157(3):474480. [PubMed: 19249417]

20. Banning AP, Pearson JF, Hall RJ. Role of balloon dilatation of the aortic valve in pregnant patients with severe aortic stenosis. Br Heart J 1993;70(6):544-545. PMCID: 1025387. [PubMed: 8280520]

21. Myerson SG, Mitchell AR, Ormerod OJ, Banning AP. What is the role of balloon dilatation for severe aortic stenosis during pregnancy? J Heart Valve Dis 2005;14(2):147-150. [PubMed: 15792172]

22. Drenthen W, Pieper PG, Roos-Hesselink JW, Schmidt AC, Mulder BJ, van Dijk AP, et al. Noncardiac complications during pregnancy in women with isolated congenital pulmonary valvar stenosis. Heart 2006;92(12):1838-1843. PMCID: 1861275. [PubMed: 16818485]

23. Deal K, Wooley CF. Coarctation of the aorta and pregnancy. Ann Intern Med 1973;78(5):706-710. [PubMed: 4711776] 
24. Expert consensus document on management of cardiovascular diseases during pregnancy. Eur Heart J 2003;24(8):761-781. [PubMed: 12800857]

25. Beauchesne LM, Connolly HM, Ammash NM, Warnes CA. Coarctation of the aorta: outcome of pregnancy. J Am Coll Cardiol 2001;38(6):1728-1733. [PubMed: 11704388]

26. Yap SC, Drenthen W, Meijboom FJ, Moons P, Mulder BJ, Vliegen HW, et al. Comparison of pregnancy outcomes in women with repaired versus unrepaired atrial septal defect. BJOG 2009;116(12):1593-1601. [PubMed: 19681849]

27. Yap SC, Drenthen W, Pieper PG, Moons P, Mulder BJ, Vliegen HW, et al. Pregnancy outcome in women with repaired versus unrepaired isolated ventricular septal defect. BJOG. 2010

28. Yentis SM, Steer PJ, Plaat F. Eisenmenger's syndrome in pregnancy: maternal and fetal mortality in the 1990s. Br J Obstet Gynaecol 1998;105(8):921-922. [PubMed: 9746388]

29. Presbitero P, Somerville J, Stone S, Aruta E, Spiegelhalter D, Rabajoli F. Pregnancy in cyanotic congenital heart disease. Outcome of mother and fetus. Circulation 1994;89(6):2673-2676. [PubMed: 8205680]

30. Apitz C, Webb GD, Redington AN. Tetralogy of Fallot. Lancet 2009;374(9699):1462-1471. [PubMed: 19683809]

31. Veldtman GR, Connolly HM, Grogan M, Ammash NM, Warnes CA. Outcomes of pregnancy in women with tetralogy of Fallot. J Am Coll Cardiol 2004;44(1):174-180. [PubMed: 15234429]

32. Drenthen W, Pieper PG, Roos-Hesselink JW, van Lottum WA, Voors AA, Mulder BJ, et al. Outcome of pregnancy in women with congenital heart disease: a literature review. J Am Coll Cardiol 2007;49(24):2303-2311. [PubMed: 17572244]

33. Gelson E, Gatzoulis M, Steer PJ, Lupton M, Johnson M. Tetralogy of Fallot: maternal and neonatal outcomes. BJOG 2008;115(3):398-402. [PubMed: 18190378]

34. Pedersen LM, Pedersen TA, Ravn HB, Hjortdal VE. Outcomes of pregnancy in women with tetralogy of Fallot. Cardiol Young 2008;18(4):423-429. [PubMed: 18559134]

35. Khairy P, Ouyang DW, Fernandes SM, Lee-Parritz A, Economy KE, Landzberg MJ. Pregnancy outcomes in women with congenital heart disease. Circulation 2006;113(4):517-524. [PubMed: 16449731]

36. Drenthen W, Pieper PG, van der Tuuk K, Roos-Hesselink JW, Voors AA, Mostert B, et al. Cardiac complications relating to pregnancy and recurrence of disease in the offspring of women with atrioventricular septal defects. Eur Heart J 2005;26(23):2581-2587. [PubMed: 16107486]

37. Ploeg M, Drenthen W, van Dijk A, Pieper PG. Successful pregnancy after an arterial switch procedure for complete transposition of the great arteries. BJOG 2006;113(2):243-244. [PubMed: 16412005]

38. Drenthen W, Pieper PG, Roos-Hesselink JW, Zoon N, Voors AA, Mulder BJ, et al. Fertility, pregnancy, and delivery after biventricular repair for pulmonary atresia with an intact ventricular septum. Am J Cardiol 2006;98(2):259-261. [PubMed: 16828604]

39. Canobbio MM, Mair DD, van der Velde M, Koos BJ. Pregnancy outcomes after the Fontan repair. J Am Coll Cardiol 1996;28(3):763-767. [PubMed: 8772769]

40. Lipscomb KJ, Smith JC, Clarke B, Donnai P, Harris R. Outcome of pregnancy in women with Marfan's syndrome. Br J Obstet Gynaecol 1997;104(2):201-206. [PubMed: 9070139]

41. Rossiter JP, Repke JT, Morales AJ, Murphy EA, Pyeritz RE. A prospective longitudinal evaluation of pregnancy in the Marfan syndrome. Am J Obstet Gynecol 1995;173(5):1599-1606. [PubMed: 7503207]

42. Gutman G, Baris HN, Hirsch R, Mandel D, Yaron Y, Lessing JB, et al. Loeys-Dietz syndrome in pregnancy: a case description and report of a novel mutation. Fetal Diagn Ther 2009;26(1):35-37. [PubMed: 19816028]

43. Vitale N, De Feo M, De Santo LS, Pollice A, Tedesco N, Cotrufo M. Dose-dependent fetal complications of warfarin in pregnant women with mechanical heart valves. J Am Coll Cardiol 1999;33(6):1637-1641. [PubMed: 10334435]

44. Salazar E, Izaguirre R, Verdejo J, Mutchinick O. Failure of adjusted doses of subcutaneous heparin to prevent thromboembolic phenomena in pregnant patients with mechanical cardiac valve prostheses. J Am Coll Cardiol 1996;27(7):1698-1703. [PubMed: 8636556] 
45. Hanania G. Management of anticoagulants during pregnancy. Heart 2001;86(2):125-126. PMCID: 1729872. [PubMed: 11454818]

46. Wilson W, Taubert KA, Gewitz M, Lockhart PB, Baddour LM, Levison M, et al. Prevention of infective endocarditis: guidelines from the American Heart Association: a guideline from the American Heart Association Rheumatic Fever, Endocarditis and Kawasaki Disease Committee, Council on Cardiovascular Disease in the Young, and the Council on Clinical Cardiology, Council on Cardiovascular Surgery and Anesthesia, and the Quality of Care and Outcomes Research Interdisciplinary Working Group. J Am Dent Assoc 2007;138(6):739-745. 47-60. [PubMed: 17545263] 


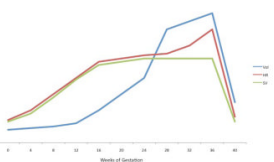

Figure 1.

Qualitative representation of the hemodynamic changes during pregnancy by week of gestation. Vol, plasma volume; HR, heart rate; SV, stroke volume. 
Table 1

CHD and Maternal Risk in Pregnancy

\author{
High risk of complications or death \\ Pulmonary hypertension \\ Eisenmenger Syndrome \\ Coarctation of the aorta, uncorrected with proximal aortic dilation \\ Marfan syndrome with aortic root diameter $>4.5 \mathrm{~cm}$ \\ Severe symptomatic aortic stenosis \\ Single ventricle with poor systolic function (with or without Fontan) \\ Moderate risk of complications (5-15\%) \\ Unrepaired cyanotic defects \\ Systemic right ventricle (d-TGA, 1-TGA) \\ Well-functioning Fontan circulation \\ Palliated Tetralogy of Fallot with severe pulmonic regurgitation and RV dysfunction \\ Low risk of complications $(<1 \%)$ \\ Isolated ASD, repaired or unrepaired \\ Isolated VSD, repaired or unrepaired \\ Coarctation, repaired with normal proximal aortic size \\ Repaired Tetralogy of Fallot with normal RV function and competent pulmonic valve
}

